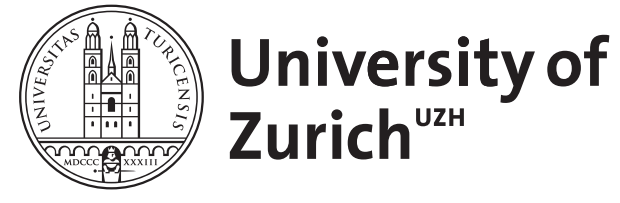

\title{
Sokrates: Tugend ist Wissen
}

Ferber, Rafael

\begin{abstract}
The article examines the Socratic principle that (1) virtue is knowledge and its corollary that (2) nobody errs voluntarily. It tries to show (I) that both principles are paradoxa, i.e. from a phenomenological point of view, they seem to be false; (II) that nevertheless the Platonic Socrates accepts both principles as true; and finally (III) that these principles are analytical truths a priori which enlarge our knowledge, but can only be understood if an individual finds them in him- or herself.
\end{abstract}

Posted at the Zurich Open Repository and Archive, University of Zurich ZORA URL: https://doi.org/10.5167/uzh-77612

Journal Article

Published Version

Originally published at:

Ferber, Rafael (1991). Sokrates: Tugend ist Wissen. Elenchos, 11:39-66. 
RAFAEL FERBER

SOKRATES: TUGEND IST WISSEN

\section{Estratto da:}

ELENCHOS

Rivista di studi sul pensiero antico

Anno XII - 1991

Fascicolo 1



BIBLIOPOLIS 
RAfael Ferber

\section{SOKRATES: TUGEND IST WISSEN}

Die zur Kennzeichnung der sokratischen Ethik verwendete These "Tugend ist Wissen" besagt: Tugend ist insofern Wissen, als das Wissen um die Tugend das Tun der Tugend impliziert. Durch Umkehrung ergibt sich, dass das Nichtwissen um die Tugend das Nichttun zur Folge hat. Fehlt man nun infolge von Unwissenheit, so nicht deshalb, weil man fehlen will, sondern unfreiwillig. Daraus ergibt sich als Korollar zum Satz "Tugend ist Wissen": "Niemand fehlt freiwillig" (nemo sua sponte peccat). Die beiden Leitsätze der sokratisch-platonischen Ethik lauten so: Das Wissen der Tugend impliziert das Tun der Tugend, und niemand fehlt freiwillig. Die Uebersetzung von arete mit Tugend wirkt dabei altertümelnd. Eher würde die Uebersetzung mit "Erfolg" uns heute näherbringen ${ }^{1}$, weshalb die sokratischen Fragen "Was ist Tugend?" und "Ist Tugend lehrbar?" für den damaligen Griechen so erheblich waren. Die sokratischen Fragen würden dann nämlich lauten: "Was ist Erfolg?" und "Ist Erfolg lehrbar?". Allerdings scheint die Uebersetzung mit Erfolg den Sinn von arete nicht adäquat wiederzugeben, insofern Erfolg zwar das Ergebnis von Arete, aber noch nicht die Haltung der Arete zu bedeuten scheint. Andere Uebersetzungen jedoch wie z.B. "Tüchtigkeit", "Exzellenz", "Bestheit" oder gar "Tucht" sind ebenfalls mit irreführenden Konnotationen belastet, so dass ich die mit "Tugend" faute de mieux

1 Auf diese Uebersetzung macht K. SEESkn, Dialogue and Discovery, A Study in Socratic Method, in Suny Series in Philosophy, ed. by R. C. Nevmle, New York 1987, SS. 118-9, aufmerksam.

ELENCHOS
1991 fasc. 1 
und als bommage an die Sprache unserer Vorfahren beibehalte. Sosehr dabei der Gebrauch von arete schon bei Plato keineswegs nur auf moralische Tugenden eingeschränkt ist - so z.B. können auch Hunde und Pferde arete haben (vgl. resp. 335 в), wenn sie z.B. gut beissen oder gut rennen -, so meint dagegen in der sokratischen These arete moralisch positives Verhalten: Nicht Rennen oder Beissen, nicht Tugend simpliciter, sondern moralische Tugend ist Wissen. Im ersten Teil dieses Aufsatzes (I) möchte ich zeigen, warum beide Leitsätze der sokratisch-platonischen Ethik Paradoxien, im zweiten Teil (II), warum beide Paradoxien ungeachtet ihres falschen Anscheins für Sokrates wahr sind und im dritten Teil (III) möchte ich sechs Thesen allgemeiner Natur zur sokratischen Verifikation der beiden Paradoxien für die frühen platonischen Dialoge zum Thema der Tugend statuieren. Wenn dabei von Sokrates gesprochen wird, so ist der platonische Sokrates des platonischen Dialogs Protagoras, 352 B-358 E, gemeint. Was dagegen der historische Sokrates tatsächlich gedacht hat, scheint aus dem Grund faktisch unbeantwortbar zu sein, weil wir keinen Text von Sokrates haben, der uns dies sagt. Wie die anderen Sokratiker, so gebrauchte auch Plato den historischen Sokrates zur Demonstration seiner eigenen Zwecke, bzw. um es mit einer schönen Formulierung A. Momigliano's zu sagen: « He (scil. Sokrates) was not a dead man whose life could be recounted. He was the guide to territories as yet unexplored ${ }^{2}$. Wiewohl dabei die Pointe des platonischen Dialoges kompliziert und $\mathrm{m}$.W. noch nicht erkannt ist ${ }^{3}$, so versuche ich doch,

2 A. Momiglino, The Development of Greek Biography, Cambridge 1971, S. 46. Vgl. grundsätzlich O. Gigon, Sokrates, Bern 1947, 1979² SS. 7-68.

3 So z.B. in den Standardwerken von P. NATORP, Platos Ideenlebre. Eine Einfübrung in den Idealismus, Hamburg 1903, SS. 10-8, 19222, SS. 519-26; A. E. TAYLOR, Plato. The Man and His Work, London 19607, SS. 257-61; P. FrIEdLÄNDER, Platon II. Die platonischen Scbriften, erste Periode, Berlin 1964 3 , SS. 26-32; W. K. C. GuThrIE, A History of Greek Philosopby, IV, Plato. The Man and His Dialogues: Earlier Period, Cambridge-London-New York-Melbourne 1975, SS. 221-35; sowie den Interpretationen von J. P. Sullivan, The Hedonism in Plato's Protagoras', "Phronesis", vi (1961) SS. 10-28; D. Gallop, The Socratic Paradox in the 'Protagoras', « Phronesis », IX (1964) SS. 117-29; A. SESONSKE, Hedonism in the 'Protagoras', * Journal of the History of Philosophy ", I (1963) SS. 73-9; G. SANTas, Plato's 'Protagoras' and explanations of weakness, « Philosophical Review ", LXXv (1966) SS. 3-33; G. VLAstos, Socrates on Acrasia, «Phoenix », Xxm (1969) SS. 71-88; ID., The Paradox sie so einfach und klar herauszuarbeiten, als ich kann. Es ist strukturell dieselbe Pointe wie die des ontologischen Gottesbeweises. Um dabei dem Missverständnis vorzubeugen, dass ich die Ansicht des Sokrates teile, möchte ich vorausschicken, dass auch ich sie wie wohl fast jedermann ausser dem platonischen Sokrates für einen Irrtum halte, allerdings für einen tiefen und ingeniösen Irrtum. Doch um zu zeigen, worin der Fehler liegt, müsste ich einen zweiten Aufsatz schreiben, so dass ich mich hier auf eine Exposition des sokratischen Irrtums mit einer knappen Indikation der Fehlerquelle beschränke. Es ist wohl eine der entscheidenden Fehlerquellen der platonischen Philosophie.

I

Bemerkenswert ist nun an diesen Leitsätzen zweierlei: (1) Plato hat an ihnen durch sein ganzes geschriebenes Werk hindurch von den frühen Dialogen bis zum Timaius und den Gesetzen impliziert, und, was den

of Socrates, in The Philosophy of Socrates, A collection of critical essays, New York 1971, SS. 1-21; C. C. W. TAYLOR, Plato. Protagoras, transl. with notes, Oxford 1976, SS. 170-200; J. C. B. GosLIng-C. C. W. TAYLOR, The Greeks on Pleasure, Oxford 1982, SS. 47-68, W. WIEIAND, Platon und die Formen des Wissens, Göttingen 1982, SS. 263-80; M. C. STOKes, Plato's Socratic Conversations. Drama and Dialetic in Three Dialogues, London 1986, SS. 349-420; G. BöHME, Der Typ Sokrates, Frankfurt 1988, SS. 79-90. Böhme schreibt zwar richtig: « Sokrates ist schon von seinen Zeitgenossen schlecht verstanden worden und das blieb im wesentlichen auch so » (S. 88). Das bleibt aber auch bei Böhme so, wenn er die sokratische These durch folgende Phänomene zu plausibilisieren sucht: « Wenn man sich seiner Traurigkeit bewusst wird, so beginnt sie schon zu verfliegen; wenn man sich seines Zornes bewusst wird, so hält man bereits inne "(S. 90). Diese Platitüde, die im übrigen noch falsch ist, da sie ja genau das auch von Sokrates nicht geleugnete Phänomen der akrasia leugnet (vgl. SS. 43-4), ist mit dem Sokrates-Paradox nicht gemeint, das Gallop richtig so charakterisierte: « it is of labyrinthine complexity, densely concentrated and elliptical at crucial points, yet presented with an air of conviction that marks is as something more than a display of sophistical fireworks" (GALLOP, art. cit., SS. 117-8). Es ist die Grundlage der platonischen Philosophie, die uns erst verstehen lässt, warum die Frage nach dem Guten so relevant wird. Irreführend ist auch Wielands Rede von einem «irrenden Willen», da für Sokrates nicht der Wille, wofür er kein Wort hat, sondern die Affekte gemessen an der Messkunst des Guten irren können. 
zweiten betrifft, auch explizit festgehalten (vgl. z.B. Prot. 345 D-E; resp. $382 \mathrm{~A}, 413 \mathrm{~A}, 589 \mathrm{E} ;$ Tim. $86 \mathrm{D}$; leg. 731 c, 860 D). Hier scheint mir einer der Gründe zu liegen, weshalb es sinnvoll ist, von einer Einheit in Platos Denken zu sprechen. (2) Beide Leitsätze scheinen "phänomenal" falsch zu sein. Sie widersprechen dem "Phänomen" der Willensschwäche und laufen insofern auch unserer Meinung (doxa) zuwider (para), dass wir willensschwach werden können. Wir dürfen sie so im ursprünglichen Wortsinne als paradoxa bezeichnen. Wenn wir wissen, was Tugend ist, so heisst das noch nicht, dass wir sie auch in die Tat umsetzen. Im Gegensatz zum Slogan "Es gibt nichts Gutes, ausser man tut es", lassen wir es gerne bei der Erkenntnis des Guten bewenden, ohne es zu tun. Ebenso: Wenn wir im moralischen Sinne fehlen, so heisst das noch nicht, dass wir nur aus Unwissenheit fehlen, sondern wir scheinen nicht nur das Gute, sondern auch das Böse wollen zu können. Das zeigt schon unsere Sprache, die nicht nur von einem "guten", sondern auch von einem "bösen" Willen spricht, nicht nur woblwollende, sondern auch übelwollende Absichten kennt. Dieses Auseinandergehen (amphisbetein) der sokratischen These mit den Phänomenen ist denn auch von Aristoteles (vgl. eth. nic. H 2. 1145 b 27-28) bis heute immer wieder beobachtet worden. Sie scheint eine Vorherbestimmtheit und Vorhersagbarkeit menschlichen Handelns nach erfolgter Erkenntnis des Guten zu involvieren, der wir nicht zustimmen würden. Eine konzise Formulierung findet sich etwa bei J. Gould:

«Socrates was wrong in supposing that if a man achieved an understanding of what justice involves, he would necessarily become just in behaviour; since the whole problem of choice intervenes between knowledge and action ${ }^{4}$.

Aus einer theoretischen Prämisse wie "Ich weiss, was Gerechtigkeit ist" folgt noch nicht die praktische Konklusion "Ich verhalte mich gerecht". Denn man kann die theoretische Prämisse wohl kennen und doch die praktische Konklusion nicht ziehen, da die theoretische Prämisse

4 J. Gould, The Development of Plato's Ethics, New York 1955, S. 6, zitiert in G. X. SANTAS, Socrates. Philosopby in Plato's Early Dialogues, London-BostonHenley 1979, S. 184 allenfalls ein Vernunftgrund, aber noch keine Wirkursache bzw. noch kein Motiv des Handelns ist. Doch sollte sie auch ein "notwendiges" Motiv sein, so noch kein binreichendes. Das Phänomen ist uns allen vertraut und vielleicht schon von Euripides im Hippolytos gegen Sokrates formuliert worden:

«Das Rechte wissen und erkennen wir, führen es aber nicht aus, die einen infolge Faulheit, die anderen, weil sie eine andere Lust anstatt des Schönen vorziehen $»(380-383)^{5}$.

Beim Apostel Paulus lesen wir: «Denn das Gute, das ich will, das tue ich nicht, sondern das Böse, das ich nicht will, das tue ich» (Rom. 7, 19). Video meliora, proboque, deteriora sequor ist ein bekanntes lateinisches, Le matin je fais des projets et le soir des sottises ein bekanntes französisches, knowing the better and doing the worse ein stehendes englisches und etwas wider besseres Wissen tun ein stehendes deutsches Wort. $\mathrm{K}$. Tucholsky schreibt einmal: «Der Zustand der gesamten menschlichen Moral lässt sich in zwei Sätzen zusammenfassen: We ought to. But we don't». La Rochefoucauld scheint den Kern des Problems getroffen zu haben: «Nous n'avons pas assez de force de suivre toute notre raison». Der Grund also, weshalb die sokratische These nicht zutrifft, besteht in unserem Mangel an Kraft, griechisch, in unserer akrasia. Genau diese akrasia leugnet Sokrates. Heute würden wir sagen, dass er das Faktum der Willensschwäche leugnet. Zwar hat er weder einen Ausdruck für Wille noch für Willensschwäche. Gleichwohl ist Sokrates das Phänomen der Willensschwäche in Gestalt der akrasia wohl bekannt. Nachdem er nämlich an Protagoras die Frage nach der Erkenntnis (episteme) gestellt hat, «ob du auch hierüber so denkst wie die meisten Menschen (boi polloi anthropoi) oder anders? » (352 в 2-3) ${ }^{6}$, charakterisiert er deren Ansicht so:

«Die meisten nämlich denken von der Erkenntnis so ungefähr, dass sie nichts Starkes, nichts Leitendes und Beherrschendes ist; und sie achten sie auch gar nicht als ein solches, sondern meinen, dass oft, wenn auch Erkenntnis im Menschen ist, sie ihn doch nicht beherrscht, sondern

5 Uebersetzt vom Verfasser.

${ }^{6}$ Die Uebersetzung folgt, wo, nicht anders vermerkt, derjenigen F. Schleiermachers. 
irgend sonst etwas bald der Zorn, bald die Lust, bald die Unlust, manchmal die Liebe, oft auch die Furcht, so dass sie offenbar von der Erkenntnis denken wie von einem elenden Wicht (andrapodou), dass sie sich von allem anderen herumzerren lässt » (Prot. 352 B-C).

Sokrates spricht hier etwas despektierlich von der Ansicht der meisten Menschen. Danach unterliegt nicht die Meinung, sondern die Erkenntnis (episteme) einer keineswegs erschöpfenden Liste von fünf Affekten, dem Zorn, der Lust, der Unlust, der Liebe und der Furcht. Wir können sie die akrasia-These unserer Erkenntnis nennen. Es handelt sich hier natürlich um eine spezifisch philosophische These, die D. Hume in der Neuzeit mit dem Slogan «Die Vernunft ist nur der Sklave der Affekte ${ }^{7}$ zur Prominenz gebracht hat. Dabei fügt $D$. Hume noch in einer eigenartigen Ueberschreitung des Humeschen Gesetzes von der Unableitbarkeit des Sollens aus dem Sein hinzu « und soll es sein $»^{8}$. Demgegenüber behauptet Sokrates in Form einer rhetorischen Frage respektvoller die kratos-These der Erkenntnis:

«Dünkt nun auch dich so etwas von ihr, oder vielmehr, sie sei etwas Schönes, das wohl den Menschen regiere, und wenn einer Gutes und Böses erkannt habe, werde er von nichts anderem mehr gezwungen werden, irgend etwas anderes zu tun, als was seine Erkenntnis ihm befiehlt, sondern die richtige Einsicht (phronesis) sei stark genug, dem Menschen durchzuhelfen? » (Prot. $352 \mathrm{c}$ ).

Sokrates bringt den professionellen Sophisten Protagoras leicht zu dem Zugeständnis der kratos-These der Erkenntnis, ohne dass dieser dabei schon die Konsequenzen seiner These - die Lejugnung der akrasia - vorwegzunehmen vermöchte:

«So dünkt es mich, antwortete er, wie du jetzt sagst, Sokrates, und zudem wäre es, wenn für irgendeinen anderen, gewiss auch für mich unziemlich zu behaupten, dass Weisheit (sophian) und Erkenntnis (epistemen) nicht das Mächtigste (kratiston) wäre unter allem Menschlichen» (Prot. 352 C-D).

7 D. Hume, Ein Traktat über menschliche Natur, I-II, Deutsche mit Anmerkungen und Register von Th. Lipps, Hamburg 1973, II, S. 153.

8 Ibid.
Als paradigmatischen Fall für das Ueberwundenwerden durch die Affekte stellt Sokrates den Affekt der Lust hin. Um die kratos-These zu beweisen, behandelt er diesen Affekt pars pro toto. Protagoras als der Verächter der Menge, die er allerdings - ohne es zu merken - selber in einer sophisticated Version repräsentiert, möchte sich auf eine Widerlegung zuerst gar nicht einlassen: «Sagen doch wohl, sprach er, die Leute, o Sokrates, noch viel anderes Unrichtiges » (Prot. 352 E). Auf die Aufforderung des Sokrates hin « die Leute zu überreden und zu belehren, was für ein Zustand das ist, was sie nennen von der Lust überwunden werden und um deswillen das Bessere nicht tun, denn erkannt habe man es ja” (Prot. 352 E-353 A), meint er gar:

«Aber o Sokrates, sagte Protagoras, was sollen wir denn die Meinung der Leute in Betrachtung ziehen, welche sagen, was ihnen einfällt? " (Prot. $353 \mathrm{~A})$.

Das heisst: Protagoras möchte und kann nicht über seine kratos-These Rechenschaft ablegen, sondern ist das prototypische Beispiel für denjenigen, der sagt, was ihm einfällt, ohne Rechenschaft zu geben. Indem er die Menge kritisiert, macht er selber den Fehler, den er bei Menge kritisiert. Im Gewande des Verächters der Menge ist Protagoras der Sprecher der Menge. So zeichnet sich schon hier die innere Verwandtschaft zwischen der Sophistik und der Meinung der Menge ab.

Wie andere Dialogpartner in anderen frühen Dialogen will Protagoras gar nicht wissen, was er eigentlich denkt. Um das wissen zu wollen, braucht es freilich Tapferkeit (vgl. Men. 81 D). Erst die Erinnerung des Sokrates, dass Protagoras Erläuterung etwas helfen wird, um zu entdecken, wie sich die Tapferkeit zu den übrigen Teilen der Tugend verhalte (vgl. Prat. 353 A-B), bringt Protagoras dazu, Sokrates weiter zu folgen (vgl. Prot. 353 в). Denn im Unterschied zu Euthyphro, Meno, Critias und Alkibiades; die quasi wegrennen vor der Entdeckung dessen, was sie selber denken', weicht Protagoras Sokrates nicht einfach feige aus. Freilich tut er das nur in der Illusion, er sei der wissende Lehrer der Menge. Der Verlauf der Belehrung erweist Protagoras selbst als den Belehrten. Doch erst durch die scheinbare Belehrung der Menge entdeckt

9 Darauf macht SEESKIN, op. cit., S. 6, aufmerksam. 
Protagoras, was er eigentlich mit der kratos-These der Erkenntnis selber denkt. Erst durch die "illusionäre" Belehrung der "anderen" vollzieht er realiter das Gebot delphischen Gottes: Gnothi sauton, das bekanntlich für Sokrates das erste Gebot der Ethik bzw. das erste Gebot von dessen «Diesseitsevangelium» (H. Maier) geworden ist ${ }^{10}$. Ich rekonstruiere den Gang dieser protagoreischen Selbsterkenntis in drei Schritten. Wir dürfen annehmen, dass es Plato mit der Publikation des Dialogs auch um die Selbsterkenntnis des Hörers bzw. Lesers geht.

\section{II}

(a) Zuerst geben Protagoras und Sokrates eine Explikation des Explikandums, von der Lust überwunden zu werden. Dazu liefern sie Beispiele:

"Noch einmal also, sprach ich, wenn sie uns fragten: Wie erklärt ihr also das, was wir nannten zu schwach sein gegen die Lust? So würde ich zu ihnen sagen: Hört denn! Protagoras und ich, wir wollen versuchen, es euch zu erklären. Ihr meint doch darunter nichts anderes, als was euch in solchen Dingen begegnet, wie dass ihr oft von Speise und Trank und Wollust als dem Angenehmen bezwungen, wiewohl ihr wisst (gignoskontes) dass es schlecht (ponera) ist, es dennoch tut? » (Prot. $353 \mathrm{c}$ ).

Auch die Menge weiss, dass diese Dinge manchmal schlecht sind, «weil sie diese Lust für den Augenblick gewähren, und also jedes für sich angenehm sind, [...] in der folgenden Zeit Krankheit und Mangel herbeiführen und viel anderes der Art bewirken? » (Prot. 353 C-D). Umgekehrt sagen sie, dass manches Schmerzliche wie «die anstrengenden Leibesübungen, die Feldzüge, die Behandlungen der Aerzte mit Brennen und Schneiden, Arzneinehmen und Fasten" gut sind, «weil sie für den Augenblick die heftigsten Qualen und Schmerzen verursachen, [...] in der Folge Gesundheit daraus entsteht und Wohlbefinden des Körpers und

10 Vgl. dazu C. RrtTer, Sokrates, Tübingen 1931, S. 57 Anm. 86, der den Ausdruck von $\mathrm{H}$. MAIER, Sokrates, sein Werk und seine gescblicbtliche Stellung, Tübingen 1913, übernimmt. Richtig schreibt Ritter: «Die Pflicht der Selbstprüfung aber lässt sich so tief und ernst fassen, dass sie wirklich die Summe aller Pflichten in sich schliesst » (S. 56).
Rettung der Staaten und sonst Herrschaft und Reichtum? 》(Prot. 354 в). Kurz: Angenehme Dinge werden dann schlecht genannt, wenn sie langfristig Schmerz, unangenehme Dinge aber dann gut, wenn sie langfristig Lust verursachen. Die imaginierte Mehrheit der Menschen stimmt dieser Explikation ihres "linguistischen" Verhaltens zu (vgl. Prot. $354 \mathrm{c} \mathrm{1,}$ 353 с 9, 353 e 4, 354 A 3-4, 354 в 1). So erreichen Sokrates und Protagoras von ihnen leicht das Zugeständnis des Hedonismus:

«Sind also diese Dinge aus einer anderen Ursache gut, als weil sie in Lust endigen und in der Unlust Abwendung und Vertreibung? Oder hab ihr ein anderes Ziel anzugeben, in Beziehung auf welches ihr sie gut nennt, als nun Lust oder Unlust? Ich glaube, sie werden kein anderes angeben. - Auch ich glaube es nicht, sagte Protagoras. - Also jagt ihr doch der Lust nach als dem Guten, und die Unlust flieht ihr als das Böse? Das würden sie zugeben. - So dünkte es ihn auch. - Dies also haltet ihr eigentlich für böse, die Unlust, und die Lust für gut; wenn ihr doch behauptet, das Wohlbefinden selbst sei in dem Fall böse, wenn es grössere Lust raubt, als es selbst enthält, oder grössere Unlust herbeiführt, als seine eigene Lust war. Denn wenn ihr in einer andern Hinsich das Wohlbefinden für böse hieltet und in Beziehung auf ein anderes Ziel: so würdet ihr uns das auch wohl sagen können, aber ihr werdet es nicht können. - Ich glaube auch nicht, dass sie es können, sagte Protagoras " (Prot. 354 B-D)

Die These des Hedonismus wird in den Worten formuliert: «Dies also haltet ihr eigentlich für böse, die Unlust, und die Lust für gut " (Prot. 354 c 5-6). Wiewohl Sokrates die Unterscheidung zwischen einem psychologischen und einem ethischen Hedonismus noch nicht macht, so indiziert doch der vorausgehende Satz «Also jagt ihr doch der Lust nach als dem Guten, und die Unlust flieht ihr als das Böse?» (Prot. 354 c 3-5), dass Sokrates der Menge einen psychologischen Hedonismus unterstellt ${ }^{11}$. Sokrates sagt "Also jagt ihr doch der Lust nach als dem Guten, und die Unlust flieht ihr als das Böse? », aber nicht «Also sollt ihr doch der Lust nachjagen als dem Guten, und die Unlust fliehen als das

11 Vgl. dazu Sulurvan, art. cit., SS. 19-20. Es erscheint seltsam, dass C. C. W. TAYLOR, Plato. Protagoras, cit, SS. 182-6, einen ethischen Hedonismus unterstellt, wiewohl bereits R. HACKFORTH, Hedonism in Plato's 'Protagoras', "Classical Quarterly », XXII (1928) SS. 39-42, richtig einen psychologischen Hedonismus nachweist. 
Böse?». Dies wäre die Folgerung aus einem ethischen Hedonismus, der in der Lust das ethisch Gute, in der Unlust das ethisch Schlechte sieht. Aus diesem psychologischen Hedonismus folgt aber weder logisch, dass wir die Lust als das Gute verfolgen und den Schmerz als das Böse meiden sollten, noch wird es uns von Sokrates "unterstellt". Sokrates lässt vielmehr erst im Gorgias Kallikles auch einen ethischen Hedonismus vertreten (vgl. Gorg. $494 \mathrm{c}$ ), den er aber mit zwei «kunstvoll geführten Beweisen» ${ }^{12}$ widerlegt (vgl. $495 \mathrm{C}-500 \mathrm{E}$ ). Wenn wir die Lust als das Gute verfolgen und den Schmerz als das Böse vermeiden, so tun wir damit noch nicht das, was wir - im ethischen Sinne - tun sollten. Zwar involviert moralisches Verhalten Glück, unmoralisches aber Unglück (vgl. Gorg. 470 C-479 A; resp. 612 A-621 D), Glück aber Lust und Unglück Unlust. Nicht aber involviert das Verfolgen von Lust und das Vermeiden von Schmerz schon moralisches Verhalten. Insoweit stimmt Sokrates mit dem common sense und der überwältigenden Mehrheit aller Moralphilosophen überein. Es erscheint so unabhängig von der Datierungsfrage unplausibel, dass Plato während der Abfassungszeit des Protagoras eine "hedonistische Epoche" durchlebte. Sollte aber der Gorgias gar vor dem Protagoras zu datieren sein, so erscheint es erst recht ausgeschlossen. Richtig schreibt vielmehr P. Friedländer: «Sieht man den Dialog in seiner dramatischen Lebendigkeit, so kann man die Partie nur so verstehen, dass Sokrates sich vorläufig auf diese Diskussionsebene begibt» ${ }^{13}$. (Hervorhebung vom V.) Wenn Sokrates aber im Protagoras der Menge einen psychologischen Hedonismus unterstellt, so heisst das aber auch noch nicht, dass Plato damit die Position des historischen Sokrates markiert ${ }^{14}$. Diese

12 FrIEdL ÏNDER, op. cit., I, S. 246.

13 Ibid., S. 282 Anm. 24; vgl. für einen Versuch, den Gorgias vor dem Prota-

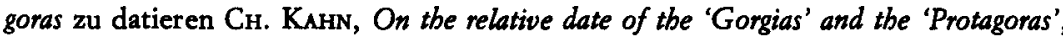
"Oxford Studies in Ancient Philosophy ", vi (1988) SS. 69-102.

14 Diese längst widerlegte Position vertreten wieder Gosurng-TAYLoR, op. cit., S. 67. Vgl. zur Literatur und Wiederlegung FrIEDLÄNDER, op. cit., S. 282 Anm. 24; GUTHRIE, op. cit., SS. 231-5, präsentiert drei verschiedene Deutungsmöglichkeiten, entscheidet sich dann aber für die auch uns richtig scheinende: « Socrates is arguing from the Sophist's own premises, his principles of relativism, empiricism and the ad boc in conduct as well as theory, illustrated in this dialogue by his little harangue on the relativity of the concept 'good' (334 a-c)» (S. 232). Die entscheidende Prämisse ist jedoch die des psychologischen Hedonismus.
Aussage wäre unverifizierbar. Er lässt vielmehr damit seinen Sokrates im Dialogkalkül eine wohlüberlegte Strategie verfolgen. Sie besteht darin, die kratos-These der Erkenntnis - Tugend ist Wissen - unter Voraussetzung der Wahrheit des psychologischen Hedonismus zu beweisen. Um das von Sokrates hier und anderswo gegenüber seinen Dialogpartners und insbesondere den Sophisten verwendete "Strategem" auch explizit zu bezeichnen: Wenn man etwas fangen will, so muss man es zuerst loslassen ${ }^{15}$."Gefangen" werden soll die Meinung der Menge und deren Sprecher Protagoras, der scheinbar wissende Lehrer der Menge. Dazu aber muss deren Meinung - der psychologische Hedonismus - voll "losgelassen", d.h. voll entfaltet werden. Dann nämlich kann Sokrates leicht die akrasia-These von innen her sich selber zerstören lassen, indem er sie der Lächerlichkeit preisgibt.

Das geschieht so: Offensichtlich ist dieser psychologische Hedonismus schon empirisch falsch, insofern auch die Menge weder jede Lust als gut verfolgt noch jede Unlust als schlecht vermeidet. Das wissen auch die Menge (vgl. Prot. 353 c) und Protagoras (vgl. Prot. 351 B-E). Deshalb versucht Sokrates die These des psychologischen Hedonismus durch eine reductio ad absurdum ihres Gegenteils zu beweisen. Wenn Wohlbefinden bzw. die Lust schlecht ist, so heisst das noch nicht, dass die Lust nicht mehr gut und somit der psychologische Hedonismus in seiner "positiven" Formulierung falsifiziert ist. Im Gegenteil: Lust ist dann schlecht, wenn sie grössere Lust raubt, als sie selbst enthält oder grössere Unlust herbeiführt, als ihre eigene Lust ist (vgl. Prot. 354 c). Lust ist also nur

15 Es handelt sich um das Strategem Nr. 16 der 36 chinesischen Strategeme herausgegeben und kommentiert von H. v. SENGER, Strategeme, Lebens-und Ueberlebenslisten der Chinesen - die berübmten 36 Strategeme aus drei Jabrtausenden, Wien $1988,1990^{5}$, SS. 293-343. Bezeichnend ist, dass diese Strategeme auch im westlichen Kulturkreis angewendet, wenn auch kaum explizit genannt werden. Dafür ist nicht nur das Neue Testament ein Beispiel, vgl. von SENGER, op. cit., SS. 366-9, 399-402, sondern auch die frühen Dialoge Platos liefern zahlreiche Beispiele für strategemisches Verhalten Sokrates' gegenüber den Sophisten. So z.B. verwendet Sokrates im Hippias Minor gegenüber dem Sophisten Hippias sowohl das Strategem 16 als auch das Strategem Nr. 10 "Hinter dem Lächeln den Dolch verbergen", von als auch das Strategem Nr. 10 "Hinter dem Lächeln den Dolch verbergen », von
SENGER, op. cit., SS. 166-75, vgl. Hippias minor, insb. 281 A-287 A. Sokrates' Vorgehen kann schwerlich anders als heimtückisch bezeichnet werden, in der Tat ein failure of love (G. Vlastos). 
insofern schlecht, als das Gute, das wir in ihr vermissen, nicht etwas anderes als Lust, sondern Lust ist. Umgekehrt: Wenn das Uebelbefinden bzw. die Unlust gut ist, so heisst das noch nicht, dass die Unlust nicht mehr schlecht und somit der psychologische Hedonismus auch in seiner "negativen" Formulierung falsifiziert ist. Im Gegenteil: Das Uebelbefinden bzw. die Unlust wird dann gut genannt, wenn es bzw. sie noch grössere Unlust, als die es bzw. sie in sich selbst hat, entfernt, oder grössere Lust, als die Unlust war, bereitet (vgl. Prot. 354 D). Unlust ist also nur insofern gut, als das Schlechte in ihr nicht etwas anderes als Unlust, sondern Unlust ist.

Aber dieser Beweis des psychologischen Hedonismus - der Position der Menge und ironischerweise auch des Protagoras - ist kein empirischer Beweis durch Beobachtung der menschlichen Psyche, sei es durch Introspektion oder durch Observation ihres Ausdrucks im Verhalten. Es ist ein logischer Beweis, der nicht Tatsachen psychologischer Natur aufdeckt, sondern die sprachlichen Bezeichnungsweisen von Folgen im Falle uneingeschränkter Maximierung von Lust und Minimierung von Unlust. Im Gegensatz nämlich zum sprachlichen Schein, wo die Ausdrücke "Lust" und "Gutes", "Schmerz" und "Schlechtes" logische unverbundene Begriffe zu bezeichnen scheinen, zeigt diese reductio, dass die These des psychologischen Hedonismus eine analytische oder begriffliche Wahrheit ist, die notwendig wahr ist. Die Bedeutung des Ausdrucks "Lust" enthält im sprachlichen Verbalten der Menge die Bedeutung des Ausdrucks "gut", und die Bedeutung des Ausdrucks "Unlust" die Bedeutung des Ausdrucks "schlecht". Deshalb kann Sokrates später die entsprechenden sprachlichen Prädikate "angenehm" und "unangenehm" durch "gut" und "schlecht" ersetzen:

«Dass dies lächerlich ist, wird euch ganz klar werden, sobald wir uns nur nicht mehr der vielerlei Namen zugleich bedienen wollen, des Angenehmen und Unangenehmen und des Guten und Schlechten, sondern da sich gezeigt hat, dass dieses nur zweierlei ist, es auch nur mit zwei Worten bezeichnen wollen, zuerst überall durch gut und schlecht und dann wieder überall durch angenehm und unangenehm " (Prot. $355 \mathrm{~B}-\mathrm{C})^{16}$.

${ }^{16}$ Kakos wird vom Vlastos jeweils mit "schlecht" und nicht mit "böse" übersetzt, um deutlich zu machen, dass der Ausdruck sowohl das moralisch als auch das aussermoralisch Schlechte bezeichnet, wohingegen "böse" vorwiegend in moralischen Kontexten verwendet wird.
Wenn wir so sagen "Die Lust bzw. das Angenehme ist gut" und "Die Unlust bzw. das Unangenehme ist schlecht", so sagen wir nicht mehr als "Die Lust ist lustvoll bzw. das Angenehme ist angenehm" und "Die Unlust ist unlustvoll bzw. das Unangenehme ist unangenehm". Wir machen nur explizit, was in der Bedeutung der Ausdrücke "Lust" und "Unlust" bereits enthalten ist. Sokrates versucht nichts anderes als zu wissen, was er sagt, wenn er spricht wie jedermann sonst - die Menge Wris sen" auf die maieutische Art und Weise zu lehren. Das heisst: Er versen" auf die maieutische Art und Wen wir bereits implizit "wissen" oder "wissen ohne es zu wissen", wenn wir unsere Wörter gebrauchen, indem er uns durch sein Fragen zwingt, es explizit zu machen: « Le plus difficile au monde est de dire en y pensant ce que tout le monde dit sans y penau monde est de sting (vot. 355 D-E). Damit konzediert er aber auch Prot. 355 D-e). Damit konzediert er aber auch die analytische Wahrheit des psychologischen Hedonismus Allerdings gilt diese analytische Wahrheit keineswegs absolut, sondern nur relativ auf sein und der Menge linguistiscbes Verhalten. Dieses "dialogrelative" Zugeständnis aber genügt Sokrates für den Beweis der kratos-These der Erkenntnis: «und dann beruht gerade hierauf der ganze Beweis (pasai bai apodeixeis) » (Prot. 354 E 6-7).

(b) Hat Protagoras als der Sprecher der Menge dieser Erklärung des psychologischen Hedonismus als einer analytischen Wahrheit zugestimmt - und dies ist der entscheidende Schachzug des Sokrates - dann hat Sokrates die Menge bzw. ihren Sprecher Protagoras auch schachmatt gesetzr. Er hat nämlich aus der Mehrheit bzw. ihrem Sprecher eine Prämisse herausgezogen, die sie für wahr halten, die jedoch die Negation der ursprünglichen These der Menge - nämlich der akrasia-These der Erkenntnis - impliziert:

«Nämlich ich sage euch, wenn sich dies so verhält, wird das nun eine lächerliche Rede, wenn ihr sagt, dass oftmals der Mensch, obgleich das Schlechte erkennend, dass es schlecht ist, es dennoch tut, unerachtet ihm frei stände, es nicht zu tun, weil er von der Lust getrieben wird und betäubt; und ihr dann auch wieder sagt, dass der Mensch, das Gute erkennend, es dennoch nicht zu tun pflegt, der augenblicklichen Lust wegen und von dieser überwunden" (Prot. 355 A-B). 
Wenn nämlich das Angenehme und Unangenehme nur verschiedene Namen für das Gute und Schlechte sind, so heisst, durch die Lust überwältigt werden, dass wir das Gute deshalb nicht tun, weil wir durch das Gute überwältigt oder überwunden werden (vgl. Prot. 355 c):

«Ist nun der, welcher uns fragt, ein Spötter, so wird er lachen und sagen, das ist doch wahrhaftig eine lächerliche Sache, was ihr da sagt, dass ein Mensch das Schlechte, indem er erkennt, dass es schlecht ist, und obwohl er es nicht tun muss, es dennoch tut, weil er vom Guten überwunden ist! »(Prot. 355 C-D).

Warum ist diese Erklärung der akrasia als Ueberwundenwerden durch das Gute "lächerlich"? D. Gallop z.B. meint, die Erklärung sei deshalb lächerlich, weil sie sich in einen expliziten Widerspruch umwandeln lasse: «The explanation is absurd in that it is transformed by means of the analysis of the expression "being overcome by pleasure" into an explicit contradiction, viz. "Though people know certain things are bad they nonetheless do them through ignorance of the fact that they are bad" "17. Doch das ist eher traurig, erklärt aber noch nicht, weshalb akrasia absurd bzw. genauer "lächerlich" ist. Eher trifft A. Graeser den Punkt: " "Jemand weiss, dass $\mathrm{X}$ schlechter ist als $\mathrm{Y}$, aber wählt gleichwohl $\mathrm{X}$, weil er meint, dass $\mathrm{X}$ besser sei als $\mathrm{Y}^{\prime \prime}{ }^{18}$. Doch warum soll das in dem von Sokrates betonten Sinne "lächerlich" sein? Das Explikandum lautet: Von der Lust überwältigt werden. Das Explikat: Vom Guten überwältigt werden. Tun wir das Gute nach erfolgter Erkenntnis des Guten dennoch nicht, weil wir vom Guten überwältigt werden, so sind diese beiden Güter - Sokrates unterscheidet zwischen Gutem und Gütern nicht - , also dasjenige, das wir nicht tun, und dasjenige, von dem wir überwältigt werden, nicht gleich gut, sondern wir werden durch ein geringeres vom Tun eines grösseren Gutes ( 355 D 8-E 1) abgehalten, so dass sich die Konsequenz ergibt: «Offenbar also, wird er sagen, meint ihr unter diesem Ueberwundenwerden, dass jemand für geringeres Gutes

${ }^{17}$ Formulierung nach TAYLOR, Plato. Protagoras, cit., S. 182. Vgl. Gallop, art. cit., SS. 120-2.

18 A. Graeser, Die Philosopbie der Antike Ir, Sophistik und Sokratik, Plato und Aristotles, in Philosophie, hg. v. W. RöD, In, München 1983, S. 103, der hier T. IRwin, Plato's Moral Theory. The Early and Middle Dialogues, Oxford 1977, S. 105 folgt. mehr Schlechtes erhält» (Prot. 355 E 2-3). Die Lächerlichkeit der akrasia nach erfolgter Substitution der Lust durch das Gute besteht so in der praktischen Dummheit oder Irrationalität, dass wir für ein geringeres Gutes ein grösseres aufgeben und dadurch mehr Schlechtes erhalten, als wenn wir das grössere Gute verwirklichten. Das ist in der Tat lächerlich. Nehmen wir z.B. ganz unsokratisch an, dass möglichst viel Geld das grösste Gut ist. Nehmen wir weiter an, dass wir unter den künstlichen Bedingungen einer Lotterie für denselben Einsatz entweder eine Million oder 1000 Franken gewinnen könnten. Dann scheint es dumm oder irrational, nicht auf eine Million zu setzen. Wir würden nämlich im Sinne des gewöhnlichen Sprachgebrauchs dumm oder irrational handeln, als wir - freie Wahl garantiert - nicht das geeignete Mittel wählen, um unser Ziel zu erreichen, nämlich möglichst viel Geld. Wenn wir aber akratisch handeln, so verhalten wir uns nach Sokrates ständig so dumm bzw. unwissend. Die Unterscheidung nämlich zwischen einem geringeren und einem grösseren Guten ist nicht durch blosse Meinung möglich, die uns der "Gewalt des Scheins" (Prot. 356 D 4) bzw. einer perspektivischen Verzerrung 19 ausliefern würde, die uns das «augenblicklich Angenehme» (Prot.356 A 6) grösser erscheinen liesse als das «für die künftige Zeit Angenehme » (Prot. 356 A 6). Sie ist nur durch die richtige Erkenntnis möglich, welches der beiden Güter das grössere und welches das geringere ist. Für diesen Zweck konzipiert Sokrates in Analogie zur Messkunst geometrischer Grössen eine Messkunst des Guten (vgl. Prot. 356 A-357 A). Nur Messung nämlich kann die notwendige Unterscheidung zwischen einem grösseren und einem geringeren Guten exakt vornehmen, wozu A. E. Taylor treffend bemerkt: «Errors of conduct are thus on the same level as false estimates of number, size, and weight $»^{20}$. Messung ist aber auch eine Kunst und Erkenntnis:

«Gut, ihr Leute. Da sich nun aber gezeigt hat, dass die Erhaltung unseres Lebens (soteria tou biou) auf der richtigen Auswahl von Lust und Unlust beruht, der mehreren und der wenigeren, grösseren und kleineren sowohl nahen als fernen: zeigt sich zuerst nicht auch diese als ein Messen, da sie Ueberschuss, Untermass und Gleichheit gegenseitig zu untersuchen

19 Graeser, op. cit., S. 104

20 A. E. TAYLOR, Plato. The Man cit., S. 259. 
hat? Notwendig ja. - Und wenn sie ein Messen ist, so ist sie notwendig eine Kunst und Erkenntnis? - Dem werden sie beistimmen. - Was für eine Kunst und Erkenntnis sie nun sein wird, wollen wir hernach sehen, dass es aber eine Erkenntnis ist, soviel ist jetzt hinreichend zu dem Beweise, den ich und Protagoras zu führen haben über das, wonach ihr uns gefragt habt » (Prot. 357 A-C).

Mit «Erhaltung unseres Lebens (soteria tou biou)» meint Sokrates nicht nur die Erhaltung unseres Ueberlebens, sondern auch die unseres guten Lebens, unseres Wohlbefindens (eu prattein) (vgl. Prot. 356 D 1). Die Erhaltung unseres Wohlbefindens beruht auf der richtigen Auswahl von Lust und Unlust. Diese aber ist ein Messen. Messen ist eine Kunst und Erkenntnis. Die Art und Weise dieser Kunst und Erkenntnis wird hier in der ersten Aussparungsstelle des platonischen Werkes ${ }^{21}$ nicht erwähnt. Es genügt das Faktum. Das Faktum aber hat eine Konsequenz. Ist nämlich die richtige Auswahl von Lust und Unlust eine (richtige) Erkenntnis, so die falsche eine falsche. Das Explikat des Explikandums "Von der Lust überwunden werden", nämlich "Vom Guten überwunden werden" unterscheidet sich so nicht nur dadurch vom Explikandum, dass es statt "Lust" "Gutes" sagt und den unbewussten Hedonismus der Menge bewusst macht. Es unterscheidet sich auch dadurch, dass es die in der Lust unbewusste kognitive Dimension zum Vorschein bringt. Wenn wir von der Lust überwältigt werden, so nur deshalb, weil wir sie für etwas Gutes im Sinne einer analytischen Wahrheit balten. Indem wir von der Lust als dem Guten überwältigt werden, so von der in der Lust qua Gutem enthaltenen kognitiven Dimension. Sokrates antizipiert so einen wichtigen, von den "Platonikern" des Mittelalters und der Neuzeit Augustinus und Pascal vertretenen, von den "Platonikern" des 20. Jahrhunderts M. Scheler und N. Hartmann aber auch systematisch vertieften Gedanken ${ }^{22}$,

${ }^{21}$ H. J. KRÄMER, Arete bei Platon und Aristoteles. Zum. Wesen und zur Geschichte der platonischen Ontologie, in « Abhandlungen d. Heidelberger Akademie der Wissenschaften, philos.-histor. Klasse ", Jahrgang 1959, Nr. 6, Heidelberg 1959, SS. 490-3; ID., Platone e $i$ fondamenti della metafisica, Introduzione e traduzione di G. ReAle, in Metafisica e Storia della Metafisica, I, Milano 1982, 1989³, S. 358.

$22 \mathrm{Vgl}$. M. SCHELER, Der Formalismus in der Ethik und die materiale Wertethik, Bern-München, $1966^{5}$, SS. 270-5; N. Hartmann, Ethik, Berlin-Leipzig 1926, SS. 104-7. Diese kognitive Geladenheit der Affekte impliziert noch keinen «Emotio- nämlich dass die Gefühle der Lust und Unlust wie andere Affekte keineswegs "blinde" Gefühle ohne kognitive Dimension sind, wie das D. Hume und in seiner Nachfolge die ethischen Emotivisten behaupten ${ }^{23}$. Sie haben vielmehr eine kognitive Dimension bzw. sind Formen des Wert- und Unwertsebens. Wir erkennen quasi nicht nur mit dem Verstand, sondern auch mit der Lust und der Unlust, den Affekten. Wenn wir Lust auf $\mathrm{X}$ und Unlust vor $\mathrm{Y}$ haben, so deshalb weil wir Lust auf $\mathrm{X}$ für gut und die Unlust for $Y$ für schlecht halten. Im Gegensatz also zu der von D. Hume wieder aufgenommenen These der Menge «Die Vernunft ist der Sklave der Affekte » könnte Sokrates sagen «Die Affekte sind der Sklave der Vernunft ". Dies aber nicht in dem noch unaufgeklärten, auch von Protagoras vertretenen Sinne, dass die Vernunft das "Mächtigste" ist unter allem Menschlichen (Prot. $352 \mathrm{D})$. Dies in dem tieferen Sinne, dass die Affekte selber schon eine kognitive Dimension haben, deshalb wahrheits- und falschheitsfähig und so "Sklaven" eines wahren oder falschen Urteils sind.

Doch ein solches Urteil der Affekte ist für Sokrates nicht unfehlbar. Im Falle der akrasia nämlich, wo wir von einem geringeren Guten zugunsten eines grösseren überwunden werden, handelt es sich am Masstab

nalen Apriorismus des Wertgefühls " (HarTManN, op. cit., SS. 104-6), sondern es handelt sich um ein durch Erfahrung gewonnenes emotionales Aposteriori, das auch Werttäuschungen zulässt.

${ }^{23}$ Vgl. D. HumE, op. cit., m, S. 195: « Nun sind augenscheinlich unsere Affekte, unsere Wollungen und unsere Handlungen einer solchen Uebereinstimmung oder Nichtübereinstimmung nicht fähig; sie sind ursprüngliche Tatsachen und Wirklichkeiten, in sich selbst vollendet, ohne Hinweis auf andere Affekte, Wollungen und Handlungen. Man kann also unmöglich von ihnen sagen, dass sie richtig oder falsch sind, der Vernunft entsprechen oder ihr widerstreiten ». Hume verkennt die phänomenologisch richtige Einsicht von der kognitiven Geladenheit der Affekte. Erst Analyse fördert das künstliche Phänomen eines reinen Affekts zutage. Phänomenologisch dagegen sind Affekte von Kognitionen nicht scharf zu trennen. Besonders krass wird das Phänomen der Affekte von A. J. AYER, Sprache, Wabrbeit und Logik, aus dem Englischen übersetzt und herausgegeben v. H. HerRING, Stuttgart 1970, SS. 141-51, verkannt, der dem ethischen Emotivismus wohl seine krudeste Form gegeben hat, aber andererseits dadurch auch dessen Unhaltbarkeit am deutlichsten zeigt. Dies heisst nicht, dass hier einem Kognitivismus sokratisch-platonische Prägung Dchon as Wort geredet wird. Doch hat jeder Emotivismus zuerst eine adaquatere Theorie der Affekte vorzulegen, als dies Hume und etwa Ayer getan haben. 
einer solchen projektierten Messkunst des Guten gemessen um eine falsche Erkenntnis. Die akrasia existiert so noch für Sokrates, aber nicht mehr als das Phänomen des Widerstreits zwischen Vernunft und Lust, sondern als das Phänomen des Widerspruchs zwischen zwei verschiedenen Kognitionen des Guten, einer richtigen und einer falschen. Da die falsche aber auf Unverstand beruht, so verwandelt sich der Widerstreit zwischen Vernunft und Lust in den Widerspruch zwischen dem Urteil von Vernunft und Unverstand. Die praktische Dummheit der akrasia erweist sich so als Unverstand. Damit ist die akrasia-These von innen her zerstört und der Lächerlichkeit preisgegeben:

«Wenn wir euch nun damals gleich gesagt hätten, er wäre eben Unverstand, so würdet ihr uns ausgelacht haben; jetzt aber, wenn ihr uns auslachen wolltet, müsst ihr euch selbst mit auslachen, denn ihr habt selbst eingestanden, wer bei der Wahl der Lust und Unlust, das heisst des Guten und Schlechten fehle, der fehle aus Mangel an Erkenntnis; und nicht nur an Erkenntnis, sondern noch weiter habt ihr zugegeben, dass es eine messende sei. Eine ohne Erkenntnis verfehlte Handlung aber, wisst ihr wohl selbst, wird aus Unverstand so verrichtet, so dass also dies das Zuschwachesein gegen die Lust ist, der grösste Unverstand (amathia be megiste» (Prot. 357 D-E).

Immer noch abhängig von der hedonistichen These, dass das Gute Lust ist, scheint so die kratos-These der Erkenntnis bewiesen und die akrasia-These widerlegt (vgl. Prot. 357 B-C). Denn wenn schon die Lust auf einer Erkenntnis des Guten beruht und wir im Falle des Ueberwundenwerdens von der Lust von einer Erkenntnis des Guten überwunden werden, so fällt die Gegenistanz gegen die kratos-These, nämlich die akrasia-These weg, wonach wird von der blossen Lust überwältigt werden. Es gibt zwar noch immer akrasia. Doch hat sie sich gewandelt: akrasia ist amathia oder Schwäche des Willens ist Schwäche der Vernunft. Umgekehrt lässt sich folgern: Stärke des Willens ist Stärke der Vernunft, d.h. Tugend ist Wissen. Wenn die scheinbare Gegeninstanz gegen die kratos-These - die akrasia-These auch in ihrer stärksten Formulierung - eine Bestätigungsinstanz der kratos-These ist, so ist die kratos-These gegen die akrasia-These bewiesen und die akrasia-These widerlegt. Da wir nämlich im Falle der akrasia von einer amatbia geleitet werden, so werden wir im Falle des Tuns der Tugend von einem Wissen geführt. Die schein- bar synthetische und durch Erfahrung falsifizierbare These "Tugend ist Wissen" entpuppt sich so als analytische These, die wahr ist, was kommen mag: Im Begriff des "Tuns der Tugend" ist der des "Wissens um die Tugend" schon enthalten und umgekehrt im Begriffe des "Wissens um die Tugend" der des "Tuns".

(c) Ist akrasia amathia, so lässt sich leicht das Korollar zum Paradox "Tugend ist Wissen", nämlich "Untugend ist Unwissenheit" bzw. niemand fehlt freiwillig oder geht freiwillig dem Schlechten nach, ableiten:

«Wie nun? Nennt ihr das Unverstand, falsche Meinungen zu haben und sich zu täuschen über wichtige Dinge? - Auch dem stimmten alle bei Ist es nicht auch so, dass niemand aus freier Wahl dem Schlechten nachgeht oder dem, was er für schlecht hält? Und dass das, wie es scheint, gar nicht in der Natur des Menschen liegt, dem nachgehen zu wollen, was er für schlecht hält, anstatt des Guten, wenn er aber gezwungen wird, von zwei Uebeln eins zu wählen, niemand das grössere nehmen wird, wenn er das kleinere nehmen darf? » (Prot. $358 \mathrm{C}-\mathrm{D}$ ).

Niemand geht freiwillig dem Schlechten nach bzw. demjenigen, was er dafür hält, weil man auf Grund der hier stillschweigend vorausgesetzten These des nun seiner selbst bewusst gewordenen Hedonismus nur die Lust als das (erkannte) Gute wollen, die Unlust aber als das (erkannte) Böse nicht wollen kann. Will man aber scheinbar das, was man für das Böse hält, so will man gar nicht das Böse als Böses, sondern als vermeintliches Gutes, wie das dann Thomas v. Aquin besonders klar so formuliert: "Malum nunquam amatur nisi sub ratione boni, scil. in quantum est secundum quid bonum et apprehenditur ut simpliciter bonum» (C.G.I., II 27, 1 ad loc.). In jedem Verfolgen des Schlechten liegt so eine verborgene Erkenntnis eines scheinbaren Guten, die sich im Lichte der richtigen Erkenntnis als falsch erweisen würde. Wir wollen nicht nur, wir können aus logischen Gründen nicht gegen unser Interesse handeln, weil Handeln impliziert, dass wir in unserem Interesse oder sub ratione boni handeln. Denn auch dann, wenn wir in Folge von akrasia gegen unser Interesse zu handeln scheinen, handeln wir gleichwohl in unserem, allerdings scheinbaren Interesse oder zugunsten eines scheinbaren Gutes. Deshalb geht niemand aus freier Wahl dem Schlechten nach oder dem, was er dafür bält, anstatt dem Guten. Würde aber jemand umgekehrt gezwungen, 
von zwei Uebeln eines zu wählen, so würde niemand das grössere nehmen, wenn er das kleinere nehmen darf (vgl. Prot. 358 C-D). J. Gould ist so nach sokratischen Voraussetzungen im Unrecht:

"Socrates was wrong in supposing that if a man achieved an understanding of what justice involves, he would necessarily become just in behaviour; since the whole problem of choice intervenes between knowledge and action".

Das Problem der Wahl kommt nicht zwischen Erkenntnis und Handlung, da man nach erreichter Erkenntnis des Guten nur das Gute wählen kann. Das Korollar "Niemand tut freiwillig Unrecht" impliziert so ein drittes Paradox, nämlich dass man nur das Gute wollen kann, das dann im Gorgias weiter entwickelt wird (vgl. Gorg. 467 c-468 B). Doch - aus der Retroperspektive - lässt sich schon dem Protagoras entnehmen, dass der platonische Sokrates auf eine vollständige Koinzidenz von Moralität und Rationalität, Immoralität und Irrationalität abzielt. Moralisches Handeln ist per definitionem rationales, unmoralisches Handeln dagegen per definitionem irrationales Handeln. Das moralische Individuum handelt per definitionem in Uebereinstimmung mit dem von ihm erkann ten Guten, d.h. in Uebereinstimmung mit seinem aufgeklärten Interesse. Das unmoralische Individuum dagegen kennt das Gute nicht bzw. handelt gegen sein noch nicht aufgeklärtes Interesse. Das moralische Individuum ist sozusagen eine wandelnde Identität. "Es tut, was es will". Das unmoralische Individuum dagegen ist ein wandelnder "Widerstreit". "Es tut nicht, was es will". Hier liegt auch der wohl entscheidende Grund, weshalb für Sokrates das moralische Individuum glücklich ist sogar in den schlimmsten äusseren Umständen, das unmoralische Individuum dagegen unglücklich, sogar in den besten äusseren Umständen. $\mathrm{Da}$ man nur das Gute wollen kann, deshalb lautet die Grundfrage der platonischen Philosophie: Was ist das Gute, das wir eigentlich wollen? Diese Frage wird in der Republik wenigstens annäherungsweise im Verlaufe des Sonnen-, Linien- und Höhlengleichnisses zu beantworten versucht ${ }^{24}$. Sie ist nicht zuletzt deshalb geschrieben worden, um die erwähnten Koin-

${ }^{24}$ Vgl. dazu R. Ferber, Platos Idee des Guten, St. Augustin 1984, 19892, SS. $49-166$ zidenzen gegen alle möglichen Einwände vom ersten bis zum letzten Buch zu beweisen. In der Mitte der Republik (vgl. 473 C-E) führt das SokratesParadox zum Paradox der Philosophenherrschaft bzw. zum Paradox des Philosophierens der Herrschenden, die zuerst das letztlich Gute - die Idee des Guteǹ - zu erkennen haben, um die Tugend der Gerechtigkeit erkennen und verwirklichen und glücklich werden zu können. Es ist hier nicht der Ort, auch dieses Paradox zu entwickeln, obwohl es eine konsequente Amplifikation des Sokrates-Paradoxes "Tugend ist Wissen" dartellt ${ }^{25}$. Abschliessend sei auf eine Beweisstruktur des Sokrates-Paradoxes aufmerksam gemacht. Sie lässt sich m.E. im Prinzip auch auf vier weitere Paradoxien anwenden, die der Gorgias entfaltet (4) der Tyrann tut nicht, was er will (vgl. Gorg. 466 D-469 c), (5) der Gerechte ist glücklich und der Ungerechte ist unglücklich (Gorg. $470 \mathrm{c}-471 \mathrm{D}$ ), (6) Unrecht zu leiden ist besser als Unrecht zu tun (vgl. Gorg. 473 A-476 A) und (7) nachdem man Unrecht getan hat, ist es besser bestraft zu werden, als nicht bestraft zu werden (vgl. Gorg. 477 A-480 A). Ich entwickle diese Beweisstruktur in sechs Schritten.

\section{III}

(1) Ein Paradox wird statuiert. Dieșes Paradox widerspricht der Meinung der Menge. So widerspricht das Paradox, dass Tugend Wissen ist, der Meinung, dass wir durch die Lust überwältigt werden können, die Tugend nicht zu verwirklichen. Die Aussage scheint falsifiziert zu sein. Eine Aussage, die falsifiziert werden kann bzw. deren Gegenteil wahr zu sein scheint, aber ist eine (scheinbar) synthetische Aussage.

(2) Doch diese Falsifikationsinstanz, dass wir durch die Lust überwältigt werden, wird in eine Bestätigungsinstanz verwandelt. Wenn wir überwunden werden durch die Lust, so nur deshalb, weil wir durch eines scheinbares Gutes überwunden werden. Das heisst für Sokrates: Wir werden durch einen Irrtum davon abgehalten, die Tugend zu verwirklichen. Sokrates scheint dabei anzunehmen, dass sich dasselbe mutatis mutandis

${ }^{25}$ Vgl. Ferber, op. cit., SS. 133-4. 
auch vom Ueberwundenwerden durch die Affekte des Zorns, der Unlust, der Liebe und der Furcht sagen liesse. Auch sie spiegeln uns ein scheinbares Gutes vor, so dass wir durch einen Irrtum davon abgehalten würden, die Tugend zu verwirklichen. Da so die einzige Falsifikationsinstanz der akrasia "als" akrasia wegfällt und in eine Bestätigungsinstanz verwandelt wird, so kann das Paradox "Tugend ist Wissen" als eine analytische Wahrheit behauptet werden, die durch gegenteilige Erfahrung nicht falsifiziert werden kann. Denn diese gegenteilige Erfahrung der akrasia "als" akrasia gibt es nicht. Wie im ontologischen Argument für die Existenz Gottes der bewusstgemachte Begriff des Wesens von Gott die Existenz Gottes logisch impliziert, so impliziert im Sokrates-Paradox das bewusstgemachte Wissen um die Tugend logisch das Tun der Tugend wie umgekehrt das Nichtwissen das Nichttun. Wie im ontologischen Argument der Satz "Gott ist" eine analytische Wahrheit bildet - das scheint die einfachste und tiefste Formulierung des ontologisches Argumentes -, so ist der Satz "Tugend ist Wissen" bzw. "Wissen um die Tugend impliziert das Tun der Tugend" oder umgekehrt "Nichtwissen um die Tugend impliziert das Nichtun" eine analytische Wahrheit. Dies scheint die einfachste und tiefste Formulierung des Sokrates-Paradoxes. G. Vlastos hat somit recht, wenn er meint, dass die sokratische Frage "Was ist X?" eine Begriffsanalyse erfordert ${ }^{26}$

(3) Diese analytische Wahrheit "Tugend ist Wissen" ist aber nicht nur eine Erläuterung dessen, was wir schon wissen, sondern erweitert unsere Erkenntnis. Sie ist also nicht eine blosse Tautologie, sondern lehrt uns, was wir vorher noch nicht wussten. T. Penner hat so ebenfalls recht, wenn er behauptet, dass die sokratische Antwort auf die Frage "Was ist X" nicht in das Analytisch-Synthetisch-Modell gepresst werden kann $^{27}$. Doch verkennt er den erkenntniserweiternden Charakter analytischer Sätze. Die Antwort liegt nämlich darin, dass analytische Sätze im Gegensatz zu Kants bis heute wirksam gebliebenem Irrtum nicht blosse Erläuterungssätze zu sein brauchen, sondern unsere Erkenntnis in

${ }^{26}$ G. VLastos, Platonic Studies, Princeton $1981^{2}$, SS. $410-7$, insb. $416-7$

27 Vgl. T. PEnner, The Unity of Virtue, «Philosophical Review », LXXXII (1973) SS. $35-68$, insb. $66-8$. einem genuinen Sinne erweitern können, wie das F. Brentano wiederentdeckt hat ${ }^{28}$. Heraklit mag eine Ahnung von dieser selbsterweiternden Analyzität gehabt haben, als er den tiefsinnigen Satz schrieb: «Der Seele ist ein logos eigen, der sich selbst erweitert " (D.-K. 22 B 115). Doch sind diese Paradoxien analytische Sätze, so auch nicht a posteriori, sondern a priori, d.h. vor aller Erfahrung und durch Erfahrung nicht falsifizierbar. Indem Sokrates diese Sätze gegenüber ihren Falsifikationsinstanzen immunisiert, entdeckt er auch deren nichtempirischen Charakter. Er entdeckt den durch Erfahrung nicht falsifizierbaren Charakter ethischer Grundsätze wie "Tugend ist Wissen", "Niemand tut freiwillig unrecht". Auch wenn jedermann glaubt das Gegenteil erfahren zu haben, so sind sie gleichwohl wahr. Deshalb konnte wohl Plato bis an sein Lebensende an ihnen festhalten.

(4) Diese Art des Lehrens, die scheinbar synthetische Sätze in analytische verwandelt, ist ein frühes Beispiel der sokratischen Art und Weise des maieutischen Lehrens, d.h. ein Beispiel des ekphorischen Lehrens, das explizit zu machen versucht, was wir vorher schon implizit wussten. Im Menon wird Sokrates das Lernen, das dem ekphorischen Lehren entspricht anamnesis nennen, d.h. Erinnerung an das, was wir vor der Geburt einmal gewusst haben und in der "Geometriestunde" am Beispiel des ungebildeten Sklaven vorführen (vgl. Men. 80 D-85 E). Es entspricht wohl Platos schriftstellerischem Oekonomieprinzip, auf die Explikation der anamnesis-Lehre im Protagoras zu verzichten, wiewohl kaum anzunehmen ist, dass sie ihm nicht schon im Protagoras präsent war. Die episteme, die der Sklave durch Analyse unserer Begriffe «nur aus sich selbst hervorholt » (Men. 85 D 4) kann aber nur deshalb durch anamnesis gewonnen werden, weil anamnesis unsere Erkenntnis erweitert, insofern wir nachher wissen, was wir vorher noch nicht wussten. In diesem Sinne kann der analytische Erkenntnisvorgang, wo er entdeckt wird, anamnesis genannt

${ }^{28}$ Vgl. z.B. F. Brentano, Psychologie vom empirischen Standpunkt, II, Von der Klassification der psychischen Phänomene, mit Einleitung, Anmerkungen und Register, hg. v. O. KRaus, Hamburg 1925, SS. 178-9; ID. Versuch über die Erkenntnis, aus seinem Nachlass hg. v. A. KASTIL, erweitert und neu eingeleitet v. F. MAYER-HILLEBRAND, Hamburg 1926, $1970^{2}$ 
werden. Wie nun anamnesis impliziert, dass wir bereits mit der Geburt eine gewisse "embryonale" Erkenntnis mitbekommen haben, so bedingt auch die Erkenntnis der Tugend, dass wir mit der Geburt schon eine "embryonale" Erkenntnis der Tugend besitzen. Man kann die Erkenntnis der Tugend gar nicht suchen, ohne sie schon in gewissem Sinne zu besitzen und d.h. nach dem Sokrates-Paradox: Man muss schon tugendhaft sein, um überhaupt die "Erkenntnis" der Tugend suchen zu können. Man muss z.B. die Tapferkeit haben, die Untersuchung weiterzuführen und nicht vor der Entdeckung dessen wegzulaufen, was man eigentlich denkt ${ }^{29}$. Da Protagoras diese gewisse Tapferkeit hat, deshalb kann Sokrates die Untersuchung über das Tugend-Paradox mit ihm durchführen. An dieser charakterlichen Voraussetzung der Philosophie hält Plato noch bis zum Siebten Brief (vgl. ep. vil 344 A-B) fest.

(5) Dieses sokratische Lehren im maieutisch-anamnestischen Sinne betrifft aber nicht irgendeine Spezialität, für die es Lehrer genug gibt, sondern die Frage, wie wir unser Leben führen sollen (vgl. Gorg. 500 c; resp. $352 \mathrm{D}$ ). Dafür gibt es erstaunlicherweise keinen richtigen Lehrer, wiewohl es doch wichtiger als alle Spezialkenntnisse ist. Zwar bildet es

${ }^{29}$ Vgl. SEEsKin, op. cit., SS. 17, 103-7. Vgl. auch die Bemerkung von S. PÉTREMENT, Le Dualisme chez Platon. Les Gnostiques et les Manichéens, Paris 1947: «On croit souvent que la formule: "la vertu est science" conduit à une sorte d'intellectualisme, qu' elle met le salut de l'homme à la portée de son intelligence: il suffit d'aquérir la science et l'on parviendra surement à la vertu. Mais si l'on cherche sur quoi repose cette formule, on voit qu'elle repose sur cette idée que celui qui fait le mal l'ignore, qu'il croit faire le bien. Or comment s'appliquerait-il à connaître le vrai quand il ne sait pas qu'il est dans l'erreur? La conséquence de cette idéé n'est pas qu'il suffit de chercher la vérité, comme si c'etait une chose facile, mais plutôt qu'on ne peut la chercher qu'à la condition de l'avoir. La vérité de Platon sera comme la Dieu de Pascal: 'tu ne me chercherais pas si tu ne m'avais trouvé' "(S. 85), zitiert in H. KELSEN, Die Illusion der Gerechtigkeit. Eine kritische Untersuchung der Sozialphilosopbie Platons, im Auftrag des Hans-Kelsen-Institutes aus dem Nachlass hg. v. K. RinghofER und R. Walter, Wien 1985, S. 423 Anm. 114. Doch muss man nich schon die ganze Wahrheit haben, um sie suchen zu können, sondern man muss sie in "embryonalem" Sinne baben, um sich an sie erinnern zu können, d.h. man muss schon in gewissem Sinne tugendhaft sein, um die Tugend suchen zu können. auch das Ziel der Sophisten wie z.B. des Protagoras, die Jünglinge besser zu machen (vgl. Prot. $318 \mathrm{~A}$ ). Doch sind es für Sokrates falsche Lehrer, weil sie zu wissen glauben, was Tugend ist und vorgeben, es lehren zu können, es de facto aber weder wissen noch lehren. Das sophistische Scheinwissen und Scheinlehren aber erscheint Sokrates wohl deshalb so bekämpfenswert, weil es im Sinne seines Paradoxes nicht auf den intelektuellen Bereich beschränkt ist, sondern notwendig ein moralisch falches Handeln bei Lehrern und Schülern zu Folge hat, wie dann Plato an der Ermordung des Sokrates zeigt ${ }^{30}$. Das intellektuelle Unbehagen, das im Dialogpartner des Sokrates durch dessen Fragen hervorgebracht wird, ist so nur ein Symptom von etwas Tieferem, nämlich ein Symptom der praktischen moralischen Falschheit seines Lebens. Deshalb kann das intellktuelle Unbehagen wie z.B. bei Alkibiades auch von Scham über in schwerwiegendes Missverständnis, im sokratischen elenchos ein bloss "lorisches" Verfahren ad rem zu sehen. Es handelt sich vielmehr um ein "logisches" Verfahren ad rem zu sehen. Es hehes" ad hominem. Umgekehrt "psychologisches" oder bollkommen moralisches Individuum eine können wir folgern, dass ein volkommen moralisches - so können wir vollkommene Erkenntnis der Tugend hätte. Deshalb - so kônnen wir folgern - gibt es kein vollkommen moralisches Individuum, nicht einma Sokrates, der nie zu wissen beanspruchte, was Tugend ist, sondern nur sein Nichtwissen reklamierte. Ebensowenig - so können wir folgern - kann es auch ein vollkommen glückliches Individuum geben, das nur tut, was es will, wohl aber viele unglückliche, die nicht tun, was sie wollen. Wie unsere Erkenntnis des Guten selbst in der Republik ironisch relativierte An Annaherung an das Gute bleibt und selbst der beste pbilosophalische IndiviSokrates - noch kein sophos ist, so kann auch das beste moralische Individuum nur moralisch und glücklich in einem approximativen, nie aber in einem vollkommenen Sinne sein. Der theoretische "Approximativismus" der platonischen Philosophie, wonach die Idee des Guten nur in der Annäherung durch drei Gleichnisse, aber nicht vollständig erreicht wird, wird so notwendig ergänzt durch einen praktischen, wonach das Gute nur approximativ $\mathrm{zu}$ verwirklichen ist. Wie das Schicksal des Sokrates

30 Vgl. das Höhlengleichnis, 517 A, dazu Ferber, op. cit., SS. 128-9. 
zeigt, genügt allerdings auch dieses approximative Wissen, aber definitive Nichtwissen, um moralisch korrekt handeln zu können.

(6) Diese Lehre des Sokrates hat aber im Gegensatz zu derjenigen des Protagoras nicht in einer langen Prunkrede (vgl. Prot. 320 C-328 E), sondern in einem Dialog zu erfolgen. Denn um ein moralisches Leben zu führen, muss ich im Sinne des Sokrates-Paradoxes selber die Erkenntnis der Tugend hervorbringen, die ich dann verwirkliche. Sowenig ich mein Leben durch andere leben lassen kann, so wenig kann ich andere die Erkenntnis der Tugend für mich lernen lassen. Plato schreibt so nicht nur deshalb Dialoge statt Traktate, um Sokrates' Gespräche nachzuahmen oder wegen seiner Kritik des geschriebenen Wortes im Phaidros (vgl. Phaedr. 274 D-279 c), worauf der "geschriebene Dialog" die angemessene Antwort zu sein scheint, oder, weil der Dialog der ideale "Anfang" der Philosophie ist, wie Ch. Griswold herausstellt ${ }^{31}$. Alle diese Gründe insbesondere der letzte - sind tief und wahr. Aber der entscheidende Grund scheint mir zu sein: Um moralisch zu leben, muss ich wissen, was Tugend ist. Dieses Wissen aber wird mein Tun nie bewirken, wenn ich es nicht selbst aus mir gebäre oder die "Umwandlung der Seele" (vgl. resp. 518 B-D) nicht selbst vollziehe. Das erste Gebot der sokratischen Ethik, nämlich "Erkenne dich selbst!" verlangt so nicht nur Erkenntnis meiner selbst, sondern auch durch mich selbst. Weder ist die Seele ein Gefäss, wohinein Wissen einfach abgelegt werden kann (vgl. Prot. 314 в), noch fliesst Wissen bei blossere äusserer Berührung von Lehrer und Schüler wie Wasser von einem vollen in ein leeres Gefäss über (vgl. symp. $175 \mathrm{D})$. Das Wissen muss vielmehr vom Schüler selber geboren werden. Um dieses Wissen zu gebären, braucht er eine Hebamme. Diese ist der erbarmungslose Frager Sokrates. Fragen und Antworten aber werden besser in einem Dialog als in einem Traktat dargestellt. Das moralische Ziel der frühen Dialoge verlangt so deren dialogische und d.h. die Mündlichkeit nachbildende Form. Entsprechend kann auch der Kontext der Rechtfertigung, das logon didonai, in den frühen Dialogen nicht vom Kontext

${ }^{31}$ Vgl. dazu den tiefschürfenden Aufsatz von CH. L. Griswold, Jr., Plato's Metaphilosophy: Why Plato Wrote Dialogues, in Platonic Writings-Platonic Readings, ed. by CH. L. Griswold, London 1988, SS. 143-67. der Entdeckung getrennt werden, weil die Rechtfertigung des logos nicht der Entdeckung getrennt werden, weil dir gebäre. Das heisst: Die Einheit von Erkenntnis und Tat im Paradox "Tugend ist Wissen" spiegelt sich von Erkenntnis und ter wohlbekannten Einheit von logos und ergon der frühen platonischen Dialoge. Deshalb sind sie nicht dramatisierte Traktate, sondern schen Dialoge. Deshal Seele. Es muss deshalb grundsätzlich fraglich bleiben, ob der von der Tübinger Schule gern zitierte Ausspruch G.W. Leibniz': «Si quelcun reduisoit Platon en système, il rendroit un grand service au genre humain ${ }^{32}$, tatsächlich die Intention Platos wiedergibt. Um nämlich ihr moralisches Ziel zu erreichen, brauchen sie wesentlich ihre dialogische Form.

Dass die sokratischen Dialoge dieses moralische Ziel aber nicht erreicht haben, zeigt die spätere Karriere von Menon, Alkibiades und anderen; von den Anklägern des Sokrates ganz zu schweigen ${ }^{33}$. Die sokratischen Dialoge scheinen so nicht nur ihr theoretisches Ziel, nämlich die Definition der Tugend, sondern auch ihr praktisches zu verfehlen. Gerne wird deshalb Sokrates heute als tragischer Held bezeichnet. Er dürfte wohl auch schon von Plato so empfunden worden sein, der einen Teil seines Lebens damit verbracht hat, darüber nachzudenken, warum Sokrates zum Tode verurteilt worden ist. Instinktiv dürfte es wohl auch dem modernen Leser wie Kallikles im Gorgias gehen! «Ich weiss nicht, wie mir gewissermassen gut vorkommt, was du sagst, Sokrates; es geht mir aber doch wie den meisten; ich glaube dir nicht sonderlich" (Gorg. $513 \mathrm{c}$ ). Ohne dass wir bereits G. Ch. Lichtenberg zustimmen müssten: "Ich kann nur die Oberfläche der Leute auf meine Seite bringen, ihr "Ich kann nur die Oberfläche der Leute auf meinnlichen Vergnügungen, des bin ich überzeugt, als ich lebe ", so zeigt dieses instinktive Nichtüberzeugtwerden des etwas nicht in Ordnung sein könnte. Der entscheidende Fehler scheint

32 G. W. LeIBNIz, Brief an Rémond, in Die pbilosophische Schriften v. G. Leib miz, hg. v. C. J. GerhardT, II, Berlin 1887, S. 637. Zitiert findet sich der Ausspruch z.B. bei KRÄmer, Platone e i fondamenti cit., (1982) S. 136 Anm. 1. G. Reale, Per una nuova interpretazione di Platone. Rilettura della metafisica dei grandi dialoghi all luce delle "dottrine non scritte", Milano 1984 (19895) verwendet ihn als Motto zu seiner Schrift, vgl. ibid., (1984) S. 5.

33 Vgl. SeESKIN, op. cit., SS. 13-4. 
mir dabei weniger auf der Linie des Aristoteles zu liegen, wiewohl Aristoteles mit seiner Sokrateskritik ein Meisterstück seiner Analysetechnik vorführt (vgl. eth. nic. H 3. 1145 b 21-H 5. 1147 b 19). Der entscheidende Fehler scheint mir strukturell, aber auch nur strukturell, derselbe wie derjenige des ontologischen Gottesbeweises: Wie aus der Definition des Wesens von Gott als demjenigen quo maius nibil cogitari potest (Anselm v. Canterbury) wohl folgt, dass Gott in apprehensione intellectus (Thomas v. Aquin, Summa Theologica, Pars I, Quaest. I, Art. 1), aber noch nicht realiter existiert, so folgt aus dem Wissen um das Wesen der Tugend höchstens, dass die Tugend in apprebensione intellectus, aber noch nicht realiter verwirklicht wird. Der Graben zwischen Erkenntnis und Wirklichkeit ist durch keine logische Brücke zu überschreiten, ohne in ihn zu fallen. Wurzelt in diesem philosophischen Fehler eines rein logischen Brückenschlages zwischen Denken und Wirklichkeit auch der ausschlaggebende philosophische Grund für die persönliche Tragik des Sokrates? 